\title{
Viewing of figurative paintings affects pseudoneglect as measured by line bisection
}

\author{
Andrea Ciricugno $^{1} \cdot$ Chiara Ferrari $^{2} \cdot$ Maria Luisa Rusconi $^{3} \cdot$ Zaira Cattaneo $^{1,4}$
}

Accepted: 2 September 2020 / Published online: 6 October 2020

(C) The Psychonomic Society, Inc. 2020

\begin{abstract}
Neurologically intact individuals usually show a leftward bias in spatial attention, known as pseudoneglect, likely reflecting a right-hemisphere dominance in the control of spatial attention. A leftward bias also seems to manifest when individuals are asked to provide aesthetic judgments about visual stimuli, like artworks. However, whether artwork perception affects the allocation of spatial attention has never been directly investigated. Here, we assessed whether viewing figurative paintings affects hemispheric imbalance in the control of spatial attention by asking participants to bisect a series of lines presented on a grey background, on figurative paintings or on non-artistic photographs of real-world scenes, while either simply observing or explicitly evaluating each image. In line with previous evidence, participants showed a leftward bisection bias in the baseline condition, reflecting pseudoneglect. Critically, the presence of a painting in the background (irrespective of whether an aesthetic evaluation was required or not) significantly shifted the bias further to the left compared to when lines were bisected over a grey background (baseline) or a photographed scene. This finding suggests that perception of visual art may affect the control of spatial attention, possibly tapping on right-hemisphere resources related to visuospatial exploration, regardless of reward apprehension mechanisms (at least when images do not evoke strong emotional reactions leading to polarized like/dislike judgements).
\end{abstract}

Keywords Attention: space-based · Cognitive neuroscience

\section{Introduction}

Neurologically healthy individuals typically show a slight, though reliable, leftward attentional bias in the orienting of spatial attention, known as pseudoneglect (for reviews see Brooks et al., 2014; Jewell \& McCourt, 2000), likely depending on a right hemispheric dominance for visuospatial processing, which is known to affect how individuals process visual stimuli. Indeed, different studies reported a preference to start visual exploration with a saccade to the left side of visual images (Dickinson \& Intraub, 2009; Foulsham et al.,

Zaira Cattaneo

zaira.cattaneo@unimib.it

1 IRCCS Mondino Foundation, Pavia, Italy

2 Department of Brain and Behavioral Sciences, University of Pavia, Pavia, Italy

3 Department of Human and Social Sciences, University of Bergamo, Bergamo, Italy

4 Department of Psychology, University of Milano-Bicocca, Milan, Italy
2013; Hartmann et al., 2019; Nuthmann \& Matthias, 2014) and more efficient processing of left-side stimuli as shown by faster response time for left-side targets in visual search tasks (Nicholls et al., 2017) and better memory for left-side stimuli (Della Sala et al., 2010; Dickinson \& Intraub, 2009).

A leftward bias also seems to manifest when individuals are asked to provide aesthetic judgements about visual stimuli. For instance, when required to judge the attractiveness of abstract visual patterns (including artworks), individuals tend to rely more on information provided by the left side of the stimulus (Rodway et al., 2019), a bias that according to the authors may also depend on the habitual left-to-right reading direction. Accordingly, participants showed a robust top-left bias when they were instructed to "light" a series of abstract paintings by means of a virtual flashlight in a way they perceived to be the most aesthetically pleasing (McDine et al., 2011). Furthermore, participants prefer advertisements with leftward lighting and are more likely to purchase these products than when the same products are lit from the right side (Hutchison et al., 2011).

If the leftward attentional bias seems to affect the way we look at visual artworks (McDine et al., 2011; Rodway et al., 
2019), an interesting issue is whether the reverse also happens, that is, whether being presented with an artwork affects hemispheric imbalance so as to affect an individual's attentional bias. Consistent evidence suggests that spatial attentional biases, as measured by line bisection, can be significantly affected by the concurrent presentation of both taskirrelevant low-level (e.g., Cattaneo et al., 2012; Sosa et al., 2011; Thomas et al., 2017) and high-level stimuli such as emotional cues or numbers (Armaghani et al., 2014; Cattaneo et al., 2010, 2014; Cleland \& Bull, 2015; Hausmann et al., 2016), which may differently activate the two hemispheres. Whether being presented with a painting also affects the allocation of spatial attention as measured by line bisection, due to possible different recruitment of the two hemispheres during art-viewing, has never been directly investigated. This issue is even more interesting when considering that there may be a relationship between the "default" spatial attentional bias of an individual and her/his evocative reactions to visual art (i.e., the larger the leftward bias, the higher the emotional reaction of that individual to visual art; Drago et al., 2008), possibly due to spatial attention and emotional processing both involving the right temporo-parietal region (Drago et al., 2008; but see Hatin \& Tottenham, 2016).

Whether art-viewing engages the two hemispheres to a different extent is still a matter of debate, with different processes involved in aesthetic evaluation being possibly differently lateralized in the brain. A predominant local (vs. global) analysis of the content may be responsible for the higher recruitment of the left hemisphere during preference decisions for figurative paintings, as suggested by prior studies using a divided visual field paradigm (Nadal et al., 2018; see also Coney \& Bruce, 2004), with local processing mainly involving the left hemisphere (Han et al., 2002; Yovel et al., 2001). Directing attention to aesthetic orientation seems to also specifically activate the left hemisphere, and in particular, the prefrontal cortex (Cela-Conde et al., 2004; Cupchik et al., 2009), a finding though that may also depend on righthandedness of participants (see Cela-Conde et al., 2004). Although mostly bilateral, activations during art-viewing in reward-related regions, such as the orbitofrontal cortex, insula and caudate nucleus, may, in turn, be more pronounced in the right hemisphere (Di Dio et al., 2007, 2011; Lacey et al., 2011; Vartanian \& Goel, 2004; note though that different types of reward may lead to different lateralization patterns in rewardrelated regions; see Arsalidou et al., 2020). Moreover, artviewing may particularly engage visuospatial processing, thus tapping more on right (compared to left) parietal resources (Cela-Conde et al., 2009; Cupchik et al., 2009; Di Dio et al., 2016).

In this study, we assessed whether being presented with an artwork (or with a photograph of a real-world scene) modulates hemispheric imbalance so as to affect an individual's attentional bias as measured by line bisection. Participants bisected a series of lines presented on (unfamiliar) figurative paintings, non-artistic photographs of real-world scenes, or a grey background (baseline condition). Images were of neutral emotional valence so to rule out emotional-valence-dependent hemispheric activations (e.g., Armaghani et al., 2014; Cattaneo et al., 2014). We included both artworks and photographs of real-world scenes because the former - by virtue of their artistic status - may lead to specific activations related to reward and visuospatial processing (e.g., Lacey et al., 2011; Lutz et al., 2013), possibly affecting hemispheric imbalance in the control of spatial attention. We also asked participants to either pay attention to the images or to evaluate them aesthetically, so to force in the latter case an aesthetic orientation that may drive specific brain activations. If, as suggested by prior studies using the divided visual field paradigm (Coney \& Bruce, 2004; Nadal et al., 2018), the left hemisphere is more involved than the right in aesthetic appreciation of figurative paintings, we should observe a reduction in the leftward attentional bias with paintings (but also possibly with photographs, if these also trigger in the observer a local analysis of the content). In turn, if right hemispheric resources involved in visuospatial processing on the one hand (Cupchik et al., 2009; Di Dio et al., 2016; Fairhall \& Ishai, 2008) and in reward processing on the other (Di Dio et al., 2007, 2011; Lacey et al., 2011; Vartanian \& Goel, 2004) play a major role in aesthetic viewing, then the expected leftward bias may even increase when lines appear on paintings (and on non-artistic photographs, at least when an explicit aesthetic evaluation is required for the latter).

\section{Method}

\section{Participants}

One-hundred and sixty neurologically healthy participants ( 82 females, mean age $=23.33$ years, $\mathrm{SD}=3.65$ years) with no previous formal or informal training in art, participated in the experiment (the sample size was decided on the basis of our prior study in which we tested possible hemispheric asymmetries in liking for paintings; Nadal et al., 2018). All participants were right-handed as assessed by a standard test (Oldfield, 1971). Written informed consent was obtained from all participants before the experiment. The protocol was approved by the local ethics committee, and participants were treated in line with the guidelines of the Declaration of Helsinki.

\section{Stimuli}

Thirty-six reproductions of paintings from the XVII to the XX century and 36 non-artistic full-colour photographs depicting natural scenes were used (subtending approximately $12.1^{\circ} \times$ 
$12.5^{\circ}$ of visual angle). Paintings (see the Appendix Table 2 for the complete list of artworks used; see Fig. 1 for examples) were representational artworks depicting still-life and landscapes, belonging to different artistic movements, including realism, impressionism and post-impressionism. We only included figurative paintings because they are usually more likely than abstract paintings to evoke an aesthetic reaction in artnaïve participants (e.g., Flexas et al., 2014; Vartanian \& Goel, 2004; Vessel \& Rubin, 2010). Paintings depicting human figures were not included to avoid the activation of specific neural networks devoted to human face and body processing. Although all stimuli were the work of renowned artists, we research (Cattaneo et al., 2017, 2020; Cela-Conde et al., 2004, 2009). Scenes were non-artistic photographs depicting objects and outdoor environments (urban and rural scenes), with content similar to paintings but not definable as artistic production per se (see Fig. 1d). All the photographs and some of the paintings were taken from a larger set of images used in previous works (Cela-Conde et al., 2004, 2009). Our sets of artistic (paintings) and non-artistic (photographed scenes) images were comparable in terms of average colour (i.e., hue, a dimension of colour that is readily experienced and that corresponds to the dominant wavelength in the colour), $t(70)=$ $1.46, p=.15$; brightness (i.e., overall darkness-to-lightness of

a

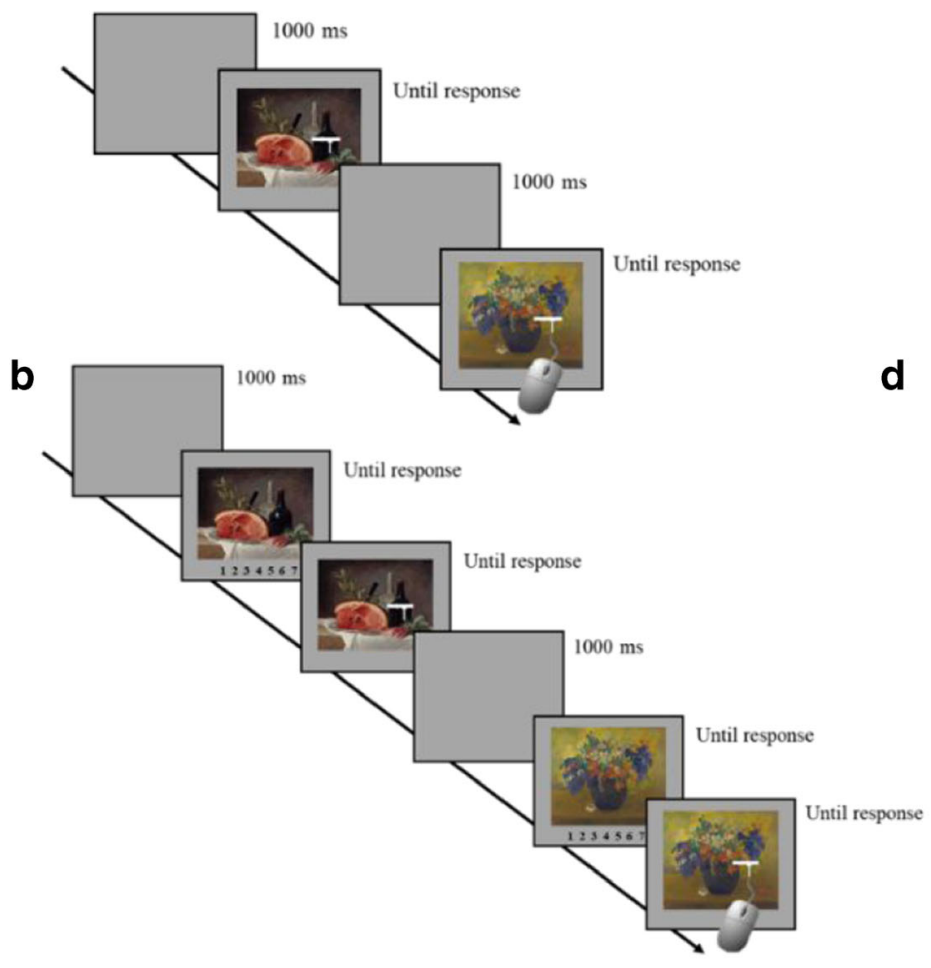
selected only relatively unknown pieces, in line with previous

a pixel's colour), $t(70)=1.44, p=.16$; and contrast (determined for each pixel by measuring the difference in colour and brightness between a pixel and the adjacent ones), $t(70)=$ $1.07, p=.29$, as we assessed using the MATLAB imageprocessing toolbox (Image Processing Toolbox Release 2017b, The MathWorks, Inc., Natick, MA, USA). Moreover, to ensure that paintings and scenes had comparable distribution of salient features that may guide the orienting of attention, we computed the saliency map for each image (see Fig. 1c) of the two stimuli sets using the computational model of Itti et al. (1998) implemented in the MATLAB SaliencyToolbox (for a detailed description of the toolbox and the saliency map computation, see Walther \& Koch, 2006). According to the 'winner-take-all' hypothesis (Itti \& Koch, 2000), for which the most salient location in the map 'wins' focal attention, we considered the first two most salient locations in each image and computed the distance between these locations and the centre of the image. The mean distance from the image centre of the first two salient locations was comparable between paintings and scenes, both along the $x$ axis, $t(70)=.27, p=79$ and $t(70)=.50, p=.62$, for the first and second salient location, respectively; and along the $y$ axis, $t(70)=.79, p=.43$ and $t(70)=.56, p=.23$, for the first and second salient location, respectively. Furthermore, in order to control for asymmetrical distribution of salient features along
C

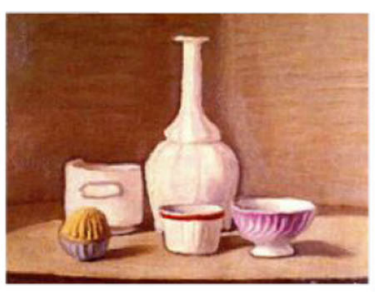

d

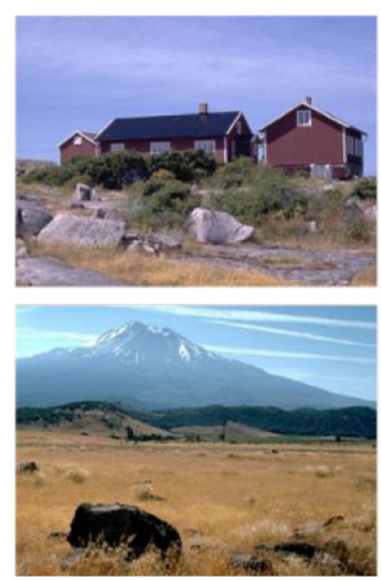

Saliency map

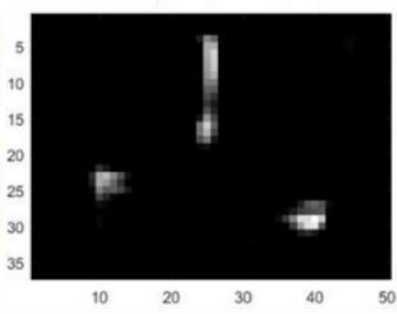

Mirror-reversed
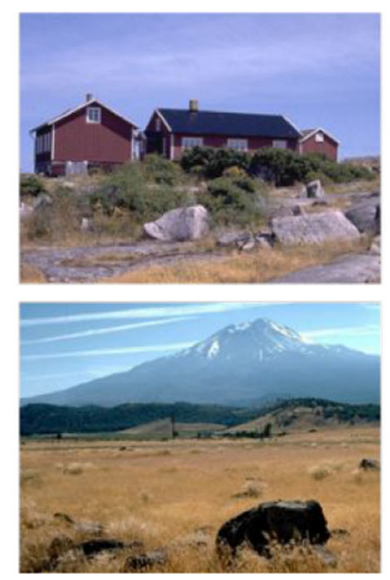

Fig. 1 Schematic overview of an experimental trial in the observation a and evaluation $\mathbf{b}$ condition with paintings presented as background. The paintings shown are Still Life with a Ham (Anne Vallayer-Coster, 1767), and Vase of Flowers (Paul Gauguin, 1896). c Example of a painting (Still
Life - Giorgio Morandi, 1946) and its saliency map. d Examples of realscenes photographs and their mirror-reversed versions. All paintings shown in this figure are free from copyright at http://www.wikiart.org/ 
the $x$ axis within each image, all stimuli were presented once in their original orientation and once left-right flipped to produce a mirror-reversed version of the same image (see Fig. 1d). Finally, all images used in the experiment were preselected so to have neutral valence, medium level of visual complexity and arousal, as further assessed in a pilot rating experiment with 60 participants $(18$ males, mean age $=26.63$ years, $\mathrm{SD}=5.24$ ), none of whom taking part in the main experiment. In the pilot rating study, evaluations for each dimension were provided on a 1-9 Likert scale $(1=$ very negative emotion/no complex at all/no arousing at all; $9=$ very positive emotion/very complex/very arousing). Paintings and scenes did not significantly differ for either emotional valence, $t(59)=1.18, p=.24$ (mean score for paintings $=4.99, \mathrm{SD}=$ 0.67 ; mean score for photographs $=4.87$; $\mathrm{SD}=0.55$ ), complexity, $t(59)<1, p=.59$ (paintings $=4.76, \mathrm{SD}=0.90$; photographs $=4.69 ; \mathrm{SD}=0.89)$, or arousal, $t(59)=1.27, p=.21$ (paintings $=5.95, \mathrm{SD}=0.95 ;$ photographs $=6.07 ; \mathrm{SD}=0.85$ ).

\section{Procedure}

Participants sat in front of a 15-in. PC screen $(1,280 \times 800$ pixels) at an approximate distance of $57 \mathrm{~cm}$, in a normally lit and silent room, and were asked to perform a computerized line bisection task. The software E-prime 2.0 (Psychology Software Tools, Inc., Pittsburgh, PA, USA) was used for stimuli presentation and data recording. Lines to be bisected could appear on a grey screen (baseline condition), on a painting or on a photograph. These viewing conditions were blocked as followed: the experiment always started with a baseline block (36 trials), followed by either the painting or photograph block (72 trials in each block; the order of these two blocks was counterbalanced across participants), and ended with a second baseline block (36 trials). Participants were pseudo-randomly (i.e., controlling for gender distribution) assigned to one of the two experimental viewing conditions: observation and evaluation. Each trial started with a blank grey screen $(1,000 \mathrm{~ms})$. In the observation condition (see Fig. 1a), this was followed by the presentation of a white line $\left(0.1^{\circ}\right.$ of visual angle thick) on the same grey screen (baseline), a painting or a real-world scene, depending on the block. Participants were asked to look at the paintings and photographs while bisecting lines. In the evaluation condition, the baseline blocks were identical to the observation condition. In turn, in the paintings and photographs blocks (see Fig. 1b), the blank screen was directly followed by the presentation of either a painting or a photograph (with no line visible), and participants were asked to indicate how much they liked each image on a 1-7 Likert scale (1: "I do not like it at all"; 7: "I like it very much") appearing at the bottom of it. No time limits were given, but participants were encouraged to give fast responses. After the response, a line appeared over the same image and participants were required to bisect it.
Paintings and photographs were always centrally presented on the screen. Lines of three different lengths $\left(3.9^{\circ}, 5.2^{\circ}\right.$ and $6.5^{\circ}$ of visual angle) were used and could appear (superimposed on the background image) in four different positions: $1.5^{\circ}$ to the left or to the right and $4.4^{\circ}$ below or above the centre of the screen. During both the observation and evaluation conditions, lines of the three different lengths appeared the same number of times in each of the four possible positions, with different combinations of line length and position being randomly generated for each participant. Participants estimated the midpoint of each line by moving the mouse with their right hand. The mouse cursor was a vertical arrow that was presented on the left or right extremity of the line at the beginning of each trial (with starting position counterbalanced across trials) and it could move only horizontally along the line. Lines remained on the screen until participants' responses; although there were no time limits, a combination of speed and accuracy was emphasized.

\section{Results}

Dependent variables were the mean percentage bisection bias and mean reaction times (RTs; recorded from the onset of the presentation of the line). The mean bisection bias (for each experimental condition) was computed by subtracting the estimated midpoint from the true centre of the line, dividing the result for the line length and multiplying the quotient by 100 (positive if bisection was to the right, negative if to the left of real midline, for a similar procedure see Cattaneo et al., 2012, 2014; Rode et al., 2006). Trials with RTs exceeding \pm 3 SD of the mean, for each participant in each condition, were excluded from the analysis (following this criterion $1.04 \%$ of trials were excluded).

\section{Mean bisection bias}

One-sample $t$-tests were conducted by comparing the mean bisection bias of each background condition (baseline, paintings, photographed scenes, collapsed for observation and evaluation) with the null set (zero - that is, the true midpoint). The analysis revealed a significant leftward bisection bias regardless of the background (see Fig. 2): baseline, $t(159)=$ 5.73, $p<.001, d=.45$; paintings, $t(159)=8.10, p<.001, d=$ .64 ; photographed scenes, $t(159)=6.38, p<.001, d=.50$. The magnitude of the bisection bias did not differ between the two baseline blocks (always performed as first and last block), $t(159)=.89, p=.37$, nor was affected by orientation manipulation either for paintings, $t(159)=.97, p=.32$, or photographed scenes $t(159)=.30, p=.74$.

A repeated-measures ANOVA with viewing condition (observation vs. evaluation) and order of presentation (paintings first followed by scenes or vice versa) as between-subjects 


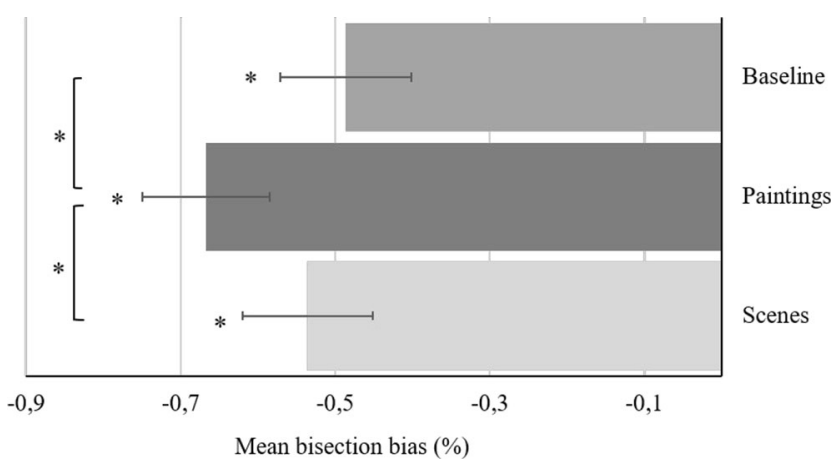

Fig. 2 Mean percentage bisection bias for the different background conditions $(\mathrm{N}=160$; collapsed across the observation and evaluation groups). Participants showed a significant leftward bias in all conditions. Pseudoneglect was significantly larger when paintings were presented as background compared to the other conditions. Error bars represent \pm SEM. Asterisks indicate significant differences between each condition and zero (the true midpoint) and between experimental conditions

variables and background (baseline, paintings, photographed scenes) as within-subjects factor, revealed a significant main effect of background, $F(2,312)=4.47, p=.012, \eta_{p}^{2}=.028$. Post hoc comparisons (Bonferroni correction applied) showed that when paintings were presented as background, participants bisected the lines further to the left compared to both photographed scenes, $t(159)=2.43, p=.048, d=.19$, and baseline, $t(159)=2.93, p=.039, d=.20$, whereas the latter two conditions did not differ from each other, $t(159)=.078, p$ $=1.0$. No other main effects or interactions reached significance (all $p s>.25$ ).

\section{Mean response latencies (RTs)}

Mean RTs are reported in Table 1. Participants were significantly faster (mean difference $=585 \mathrm{~ms}$ ) in the second baseline block compared to the first one, $t(159)=8.71, p<.001$, reflecting practice effects. A repeated-measures ANOVA with viewing condition (observation vs. evaluation) and order of presentation (paintings first followed by scenes or vice versa) as between-subjects variables and background (baseline, paintings, photographed scenes) as within-subjects factor on mean RTs showed a significant main effect of viewing condition, $F(2,312)=7.11, p=.008, \eta_{p}^{2}=.044$, a significant interaction background by viewing condition, $F(2,312)=$ $56.05, p<.001, \eta^{2}=.264$, and a significant interaction background by order, $F(2,312)=23.67, p<.001, \eta_{p}^{2}=.132$. No other main effects or interactions reached significance (all $p s$ >.09). Post hoc comparisons (Bonferroni correction applied) revealed that participants in the 'observation' group were faster in the baseline blocks compared to when paintings, $t(79)=8.45, p<.001, d=.95$, and scenes, $t(79)=6.67$, $p<.001, d=.72$, appeared as background, confirming that they took time to observe the images before bisecting. Participants of the 'evaluation' group were slower in the baseline condition compared to both the paintings, $t(79)=4.57, p<.001, d=.51$, and the photographed scenes conditions, $t(79)=4.93, p<.001$, $d=.55$ (this pattern was only apparently opposite to that in the observation condition, since RTs were recorded from the onset of the line - and not of the images, that participants of the evaluation group could freely observe before the appearance of the line). RTs in the paintings and photographed scenes blocks did not significantly differ in either the observation, $t(79)=1.78, p=.23$, or the evaluation group, $t(79)=1.03, p$ $=.12$. The significant interaction background by order was due to participants being overall slower in the block with background images (either paintings or photographed scenes) they performed as first compared to the other conditions, again likely reflecting practice effects. Specifically, participants viewing paintings first were slower with paintings compared to scenes, $t(79)=5.00 p<.001, d=.63$, and compared to baseline, $t(79)=3.97, p<.001, d=.41$; participants viewing photos first were slower with photos compared to paintings, $t(79)=3.41, p=.003, d=.59$, and to baseline, $t(79)=1.84, p$ $=.05, d=.19$.

Moreover, participants in the evaluation viewing condition did not differ in their liking judgements for paintings $(\mathrm{M}=$ 3.36 , range $=1.59-5.62)$ and photographed scenes $(\mathrm{M}=3.31$, range $=1.82-5.01), t(79)=.60, p=.71$. Response latencies in providing liking judgments were also similar for the two image categories, $t(79)=.54, p=.59$. Finally, to assess whether liking judgements influenced the magnitude of the bisection bias, we carried out a regression coefficient analysis by estimating regression coefficients individually for each

Table 1 Mean response times (RTs, ms) as a function of viewing (observation and evaluation) and background conditions (baseline, paintings, photographed scenes) recorded from the onset of the line

(observation and evaluation conditions) and from the onset of the background image to the onset of the line (evaluation condition)

\begin{tabular}{llll}
\hline & Observation & Evaluation & \\
\cline { 3 - 4 } & RTs from onset of the line & RTs from onset of the line & RTs from onset of the image to onset of the line \\
\hline Baseline & $2347(\mathrm{SD}=850)$ & $2509(\mathrm{SD}=938)$ & $2067(\mathrm{SD}=859)$ \\
Paintings & $2878(\mathrm{SD}=1106)$ & $2197(\mathrm{SD}=1004)$ & $2030(\mathrm{SD}=886)$ \\
Scenes & $2767(\mathrm{SD}=1042)$ & $2127(\mathrm{SD}=861)$ & \\
\hline
\end{tabular}


participant in the evaluation group. Specifically, we performed simple regression analyses with mean bisection bias as dependent variable and liking scores as predictor, separately for each participant. We then tested whether the extracted $\beta$ coefficients were significantly different from zero at the group level by means of a one-sample $t$-test (Lorch \& Myers, 1990; Pfister et al., 2013). The analysis revealed that the average $\beta$ coefficients of the group $(\beta=0.074)$ deviated significantly from zero, $t(79)=3.95, p<.001, d=.44$, suggesting the presence of a relationship between liking scores and bisection biases, with higher liking scores predicting more positive (i.e., rightward) bisection biases. The same pattern emerged when the analysis was performed separately for the two set of images: $t(79)=2.09, p=.04, d=.23$, for paintings $(\beta=$ $0.054)$; and $t(79)=4.9, p<.001, d=.55$, for photographed images $(\beta=0.105)$.

\section{Discussion}

In this study, we investigated whether viewing figurative paintings affects hemispheric imbalance in the control of spatial attention as measured by a line bisection task. We asked participants to bisect a series of lines presented on a grey background, on figurative paintings or on non-artistic photographs of real-world scenes, while either simply observing or explicitly evaluating each image. In line with previous evidence (for reviews see Brooks et al., 2014; Jewell \& McCourt, 2000), participants showed a leftward bisection bias in the baseline condition, reflecting pseudoneglect. Critically, the presence of a painting in the background (irrespective of whether an aesthetic evaluation was required or not) significantly shifted the bias further to the left compared to when lines were bisected over a grey background (baseline) or a photographed scene.

The increase in the leftward bisection bias induced by viewing figurative paintings (but not non-artistic photographs) may depend on activation of reward-related regions - such as the orbitofrontal cortex, insula and caudate nucleus - involved in art perception preferentially in the right hemisphere (e.g., Di Dio et al., 2011; Lacey et al., 2011; Vartanian \& Goel, 2004). Interestingly, it has been suggested that greater dopaminergic activity in the right striatum, as well as in right frontal and temporal regions, is associated with a stronger leftward attentional bias, whereas greater activity in left homologous regions is associated with an attentional bias toward the opposite direction (Tomer et al., 2013). If the viewing of paintings elicited more activation in right-hemisphere reward-related regions (Di Dio et al., 2011; Lacey et al., 2011; Vartanian \& Goel, 2004), this might have boosted the contribution of ipsilateral regions involved in the orienting of attention (Tomer et al., 2013), thus strengthening the leftward attentional bias. However, note that participants in the evaluation group assigned similar liking scores to photographs and paintings; moreover, the more they liked an image (regardless of its artistic status), the more the bisection was biased to the right, suggesting a larger recruitment of left-hemispheric resources during observation of preferred stimuli (see also Nadal et al., 2018). This discourages an interpretation of our results based on the higher rewarding potential of our selection of paintings compared to non-artistic photographs. Accordingly, asking participants to provide an explicit aesthetic evaluation did not affect bisection performance, further suggesting that focusing on aesthetic qualities of the stimuli may not be the main process underlying our results.

An alternative explanation is that viewing paintings possibly further recruited - compared to the baseline condition right parietal regions involved in visual exploration behaviour (Cela-Conde et al., 2009; Di Dio et al., 2016), thus boosting the attentional bias. Although one may object that a similar mechanism should also be at play with photographs of real scenes, there is evidence that paintings may require more visuospatial encoding than real-world scenes (e.g., Lutz et al., 2013). This may also depend on the lack of routinized exploratory strategies for artworks in art-naïve observers (like our participants), with the right hemisphere being possibly more critical than the left for exploratory processing of "novel" stimuli (see Vogt \& Magnussen, 2005). In fact, even if scenes and paintings were matched for complexity in our task and the two sets did not differ overall in their spatial composition of salient elements (salient locations in an image affecting the orienting of attention; Itti \& Koch, 2000), pictorial representations may indeed require higher-order spatial processing in order to bind object form and spatial location within the pictorial scene, as well as to link the various visual elements (e.g., Fairhall \& Ishai, 2008).

In a prior study, Nadal et al. (2018) observed higher preference for figurative paintings shortly $(150 \mathrm{~ms})$ presented in the right than in the left hemifield and hypothesized that this pattern was due to aesthetic evaluation of figurative artworks being mostly driven by local analysis of the depicted content, with local visual processing preferentially recruiting resources in the left hemisphere (Han et al., 2002; Yovel et al., 2001). Similarly, we found that observers tended to bisect the lines more rightward (reflecting a larger recruitment of the left hemisphere) the more they liked the background image, regardless the artistic status of the image and for viewing durations much longer than in Nadal et al. (2018) (more than $2 \mathrm{~s}$, see Table 1). It has been suggested that aesthetic evaluation is a dynamic process comprising an initial 'gross' and fast evaluation of the aesthetic qualities of the image (formed within the 250-750 ms after image presentation), mainly involving occipital regions, and a later more detailed aesthetic evaluation (that is formed around 1,000-1,500 ms from image onset), which involves a larger network of brain regions, including the default mode network (Cela-Conde et al., 2013; see 
also Belfi et al., 2019). Our results suggest that at this later stage viewing of paintings is associated with different processes simultaneously at play and involving the two hemispheres to a different extent: on one hand, artworks seem to recruit more right-hemisphere resources than non-artistic images for enhanced visuospatial processing (e.g., Lutz et al., 2013); on the other hand aesthetic evaluation (at least for figurative images and irrespective of their artistic status) is likely to rely mostly on left-hemisphere resources for analysis of local content (see Nadal et al., 2018).

Finally, in interpreting our results, it is important to consider that we only included emotionally neutral stimuli, with little arousing potential, thus avoiding emotional valence-related hemispheric activations that have been found to affect bisection bias (Armaghani et al., 2014; Cattaneo et al., 2014). Accordingly, our stimuli received medium liking scores (see the Likert scores provided by participants in the evaluation group). It may be that selecting stimuli eliciting more polarized rating scores in terms of liking and/or emotional reaction (also providing information about the paintings to show that they are by famous artists, e.g., Huang et al., 2011; Millis, 2001) might differently activate the two hemispheres compared to unfamiliar emotionally neutral paintings, a hypothesis that future studies may address.

In sum, our findings suggest that viewing figurative paintings affects the orienting of spatial attention, increasing the typical leftward bias shown by neurologically healthy individuals in space exploration, as measured by a line bisection task. This pattern may depend on art viewing recruiting righthemisphere resources important for visuospatial exploration of the image (Cela-Conde et al., 2009; Di Dio et al., 2016; Lutz et al., 2013), regardless of reward apprehension (at least when images do not evoke strong emotional reactions leading to polarized like/dislike judgements). Our findings may be potentially of interest also from a clinical perspective, for rehabilitation programs of spatial attentional deficits (see Guilbert et al., 2014).

Acknowledgements We are grateful to Marcos Nadal for discussing the experimental idea and helping with stimuli selection. We thank Camilo Cela-Conde and colleagues for sharing the stimuli used in Cela-Conde et al. (2004): Activation of the prefrontal cortex in the human visual aesthetic perception, PNAS, 101(16), 6321-6325. This work was supported by a PRIN Grant (2015WXAXJF) by Italian Ministry of Education, University and Research to Z.C and by Mondino Foundation "Ricerca Corrente" funds.

Open practices statement The data and materials of the experiment are available on request.

\section{Appendix}

Table 2 Painting stimuli

Alexander Kanoldt (1926) Still Life

Alexander Kanoldt (1926) Still Life IV

Alexej Von Jawlensky (1912) Landshaften

Alfred Henry Maurer (1909-1910) Still Life

Anne Vallayer-Coster (1767) Still Life with a Ham

Aureliano De Beruete Y Moret (1904) El Tajo Toledo Prado

Camille Corot (1830) Houses Near Orleans

Claude Monet (1874) The Bridge in Argenteuil

Claude Monet (1879) Still Life With Apples and Grapes

Ernst Ludwig Kirchner (1918) Frauenkirck im Winter

Ernst Ludwig Kirchner (1919-1920) Grey Cat on a Cushion

Francisco de Zurbaran (1633) Still Life

Franz Marc (1914) Small Composition II

Georgia O'Keeffe (1922) Ends of Barns

Giorgio De Chirico (1925) Metaphysical Interior

Giorgio Morandi (1946) Still Life

Giorgio Morandi (1938) Still Life

Giorgio Morandi (1952) Still Life

Henri Fantin-Latour (1866) Still Life with Flowers and Fruit

Henri Rousseau (1890) The Toll House

Henri Rousseau (1896) View of the Outskirts of Paris

Henri Rousseau (1910) Still Life with Teapot and Fruit

Jan van Goyen (1642) A Windmill by a River

Johannes Vermeer (1658-1660) The Little Street

John Constable (1822) View on the Sour

Karl Schmidt-Rottluff (1937) Blue Window

Leslie Hunter (1914-1918) Kitchen Utensilis

Marc Chagall (1970) Flowers on a Red Ground

Othon Friesz (1907) Paysage

Paul Cezanne (1876) Landscape, Study after Nature

Paul Gauguin (1896) Vase of Flowers

Philips Koninck (1649) Landscape

Pierre-Auguste Renoir (1883) Apples in a Dish

Theodore Gericault (1821) Dead Cat

Wayne Thiebaud (1920) Jackpot Machine

Wilfred Fairclough (1945-1947) High Wycombe, Buckingamshire 


\section{References}

Armaghani, S. J., Crucian, G. P., \& Heilman, K. M. (2014). The influence of emotional faces on the spatial allocation of attention. Brain and cognition, 91, 108-112.

Arsalidou, M., Vijayarajah, S., \& Sharaev, M. (2020). Basal ganglia lateralization in different types of reward. Brain Imaging and Behavior, 1-29.

Belfi, A. M., Vessel, E. A., Brielmann, A., Isik, A. I., Chatterjee, A., Leder, H., ... Starr, G. G. (2019). Dynamics of aesthetic experience are reflected in the default-mode network. Neuroimage, 188, 584597.

Brooks, J. L., Della Sala, S., \& Darling, S. (2014). Representational pseudoneglect: A review. Neuropsychology review, 24(2), 148-165.

Cattaneo, Z., Fantino, M., Tinti, C., Silvanto, J., \& Vecchi, T. (2010). Crossmodal interaction between the mental number line and peripersonal haptic space representation in sighted and blind individuals. Attention, Perception, \& Psychophysics, 72(4), 885-890.

Cattaneo, Z., Ferrari, C., Schiavi, S., Alekseichuk, I., Antal, A., \& Nadal, M. (2020). Medial prefrontal cortex involvement in aesthetic appreciation of paintings: at DCS study. Cognitive processing, 21(1), 6576.

Cattaneo, Z., Lega, C., Boehringer, J., Gallucci, M., Girelli, L., \& Carbon, C. C. (2014). Happiness takes you right: the effect of emotional stimuli on line bisection. Cognition \& emotion, 28(2), 325-344.

Cattaneo, Z., Lega, C., Vecchi, T., \& Vallar, G. (2012). Listening to white noise counteracts visual and haptic pseudoneglect. Perception, 41(11), 1395-1398.

Cattaneo, Z., Schiavi, S., Silvanto, J., \& Nadal, M. (2017). A TMS study on the contribution of visual area V5 to the perception of implied motion in art and its appreciation. Cognitive neuroscience, 8(1), 5968.

Cela-Conde, C. J., Ayala, F. J., Munar, E., Maestú, F., Nadal, M., Capó, M. A., ... Marty, G. (2009). Sex-related similarities and differences in the neural correlates of beauty. Proceedings of the National Academy of Sciences, 106(10), 3847-3852.

Cela-Conde, C. J., García-Prieto, J., Ramasco, J. J., Mirasso, C. R., Bajo, R., Munar, E., ... Maestú, F. (2013). Dynamics of brain networks in the aesthetic appreciation. Proceedings of the National Academy of Sciences, 110(Supplement 2), 10454-10461.

Cela-Conde, C. J., Marty, G., Maestú, F., Ortiz, T., Munar, E., Fernández, A., ... Quesney, F. (2004). Activation of the prefrontal cortex in the human visual aesthetic perception. Proceedings of the National Academy of Sciences, 101(16), 6321-6325.

Cleland, A. A., \& Bull, R. (2015). The role of numerical and nonnumerical cues in nonsymbolic number processing: Evidence from the line bisection task. Quarterly journal of experimental psychology, 68(9), 1844-1859.

Coney, J., \& Bruce, C. (2004). Hemispheric processes in the perception of art. Empirical Studies of the Arts, 22(2), 181-200.

Cupchik, G. C., Vartanian, O., Crawley, A., \& Mikulis, D. J. (2009). Viewing artworks: contributions of cognitive control and perceptual facilitation to aesthetic experience. Brain and cognition, 70(1), 8491.

Della Sala, S., Darling, S., \& Logie, R. H. (2010). Items on the left are better remembered. The Quarterly Journal of Experimental Psychology, 63(5), 848-855.

Di Dio, C., Ardizzi, M., Massaro, D., Di Cesare, G., Gilli, G., Marchetti, A., \& Gallese, V. (2016). Human, nature, dynamism: the effects of content and movement perception on brain activations during the aesthetic judgment of representational paintings. Frontiers in human neuroscience, 9, 705 .

Di Dio, C., Canessa, N., Cappa, S. F., \& Rizzolatti, G. (2011). Specificity of esthetic experience for artworks: an fMRI study. Frontiers in human Neuroscience, 5, 139.
Di Dio, C., Macaluso, E., \& Rizzolatti, G. (2007). The golden beauty: brain response to classical and renaissance sculptures. PloS one, 2(11).

Dickinson, C. A., \& Intraub, H. (2009). Spatial asymmetries in viewing and remembering scenes: Consequences of an attentional bias? Attention, Perception, \& Psychophysics, 71(6), 1251-1262.

Drago, V., Finney, G. R., Foster, P. S., Amengual, A., Jeong, Y., Mizuno, T., ... Heilman, K. M. (2008). Spatial-attention and emotional evocation: Line bisection performance and visual art emotional evocation. Brain and cognition, 66(2), 140-144.

Fairhall, S. L., \& Ishai, A. (2008). Neural correlates of object indeterminacy in art compositions. Consciousness and cognition, 17(3), 923932.

Flexas, A., Rosselló, J., de Miguel, P., Nadal, M., \& Munar, E. (2014). Cognitive control and unusual decisions about beauty: an fMRI study. Frontiers in human neuroscience, 8, 520.

Foulsham, T., Gray, A., Nasiopoulos, E., \& Kingstone, A. (2013). Leftward biases in picture scanning and line bisection: A gazecontingent window study. Vision research, 78, 14-25.

Guilbert, A., Clément, S., \& Moroni, C. (2014). Hearing and music in unilateral spatial neglect neuro-rehabilitation. Frontiers in psychology, 5, 1503.

Han, S., Weaver, J. A., Murray, S. O., Kang, X., Yund, E. W., \& Woods, D. L. (2002). Hemispheric asymmetry in global/local processing: effects of stimulus position and spatial frequency. Neuroimage, 17(3), 1290-1299.

Hartmann, M., Sommer, N. R., Diana, L., Müri, R. M., \& EberhardMoscicka, A. K. (2019). Further to the right: Viewing distance modulates attentional asymmetries ('pseudoneglect') during visual exploration. Brain and cognition, 129, 40-48.

Hatin, B., \& Sykes Tottenham, L. (2016). The relationship between line bisection performance and emotion processing: Where do you draw the line?. Laterality: Asymmetries of Body, Brain and Cognition, 21(4-6), 709-731.

Hausmann, M., Hodgetts, S., \& Eerola, T. (2016). Music-induced changes in functional cerebral asymmetries. Brain and cognition, 104, 5871

Huang, M., Bridge, H., Kemp, M. J., \& Parker, A. J. (2011). Human cortical activity evoked by the assignment of authenticity when viewing works of art. Frontiers in human neuroscience, 5, 134.

Hutchison, J., Thomas, N. A., \& Elias, L. (2011). Leftward lighting in advertisements increases advertisement ratings and purchase intention. Laterality: Asymmetries of Body, Brain and Cognition, 16(4), 423-432.

Itti, L., \& Koch, C. (2000). A saliency-based search mechanism for overt and covert shifts of visual attention. Vision research, 40(10-12), 1489-1506.

Itti, L., Koch, C., \& Niebur, E. (1998). A model of saliency-based visual attention for rapid scene analysis. IEEE Transactions on pattern analysis and machine intelligence, 20(11), 1254-1259.

Jewell, G., \& McCourt, M. E. (2000). Pseudoneglect: a review and metaanalysis of performance factors in line bisection tasks. Neuropsychologia, 38(1), 93-110.

Lacey, S., Hagtvedt, H., Patrick, V. M., Anderson, A., Stilla, R., Deshpande, G., ... Sathian, K. (2011). Art for reward's sake: Visual art recruits the ventral striatum. Neuroimage, 55(1), 420-433.

Lorch, R. F., \& Myers, J. L. (1990). Regression analyses of repeated measures data in cognitive research. Journal of Experimental Psychology: Learning, Memory, and Cognition, 16(1), 149.

Lutz, A., Nassehi, A., Bao, Y., Pöppel, E., Sztrókay, A., Reiser, M., ... Gutyrchik, E. (2013). Neurocognitive processing of body representations in artistic and photographic images. Neuroimage, 66, 288292.

McDine, D. A., Livingston, I. J., Thomas, N. A., \& Elias, L. J. (2011). Lateral biases in lighting of abstract artwork. Laterality, 16(3), 268279. 
Millis, K. (2001). Making meaning brings pleasure: The influence of titles on aesthetic experiences. Emotion, 1(3), 320.

Nadal, M., Schiavi, S., \& Cattaneo, Z. (2018). Hemispheric asymmetry of liking for representational and abstract paintings. Psychonomic bulletin \& review, 25(5), 1934-1942.

Nicholls, M. E., Hobson, A., Petty, J., Churches, O., \& Thomas, N. A. (2017). The effect of cerebral asymmetries and eye scanning on pseudoneglect for a visual search task. Brain and cognition, 111, 134-143.

Nuthmann, A., \& Matthias, E. (2014). Time course of pseudoneglect in scene viewing. Cortex, 52, 113-119.

Oldfield, R. C. (1971). The assessment and analysis of handedness: the Edinburgh inventory. Neuropsychologia, 9(1), 97-113.

Pfister, R., Schwarz, K. A., Carson, R., \& Janczyk, M. (2013). Easy methods for extracting individual regression slopes: Comparing SPSS, R, and Excel. Tutorials in Quantitative Methods for Psychology, 9(2), 72-78.

Rode, G., Michel, C., Rossetti, Y., Boisson, D., \& Vallar, G. (2006). Left size distortion (hyperschematia) after right brain damage. Neurology, 67(10), 1801-1808.

Rodway, P., Schepman, A., Crossley, B., \& Lee, J. (2019). A leftward perceptual asymmetry when judging the attractiveness of visual patterns. Laterality: Asymmetries of Body, Brain and Cognition, 24(1), $1-25$.

Sosa, Y., Clarke, A. M., \& McCourt, M. E. (2011). Hemifield asymmetry in the potency of exogenous auditory and visual cues. Vision research, 51(11), 1207-1215.
Thomas, N. A., Rose, W., \& Nicholls, M. E. (2017). The influence of distractors and numerical direction on mental number line bisection. Laterality: Asymmetries of Body, Brain and Cognition, 22(1), 3148.

Tomer, R., Slagter, H. A., Christian, B. T., Fox, A. S., King, C. R., Murali, D., \& Davidson, R. J. (2013). Dopamine asymmetries predict orienting bias in healthy individuals. Cerebral Cortex, 23(12), 2899-2904

Vartanian, O., \& Goel, V. (2004). Neuroanatomical correlates of aesthetic preference for paintings. Neuroreport, 15(5), 893-897.

Vessel, E. A., \& Rubin, N. (2010). Beauty and the beholder: Highly individual taste for abstract, but not real-world images. Journal of Vision, 10(2), 18-18.

Vogt, S., \& Magnussen, S. (2005). Hemispheric specialization and recognition memory for abstract and realistic pictures: A comparison of painters and laymen. Brain and Cognition, 58, 324-333.

Walther, D., \& Koch, C. (2006). Modelling attention to salient protoobjects. Neural networks, 19(9), 1395-1407.

Yovel, G., Yovel, I., \& Levy, J. (2001). Hemispheric asymmetries for global and local visual perception: effects of stimulus and task factors. Journal of Experimental Psychology: Human Perception and Performance, 27(6), 1369.

Publisher's note Springer Nature remains neutral with regard to jurisdictional claims in published maps and institutional affiliations. 